\title{
FDI und OPC UA
}

Grundsteine von Industrie 4.0

Ein wesentlicher Baustein für die Umsetzung von Industrie-4.0-Anwendungsfällen ist die flexible Vernetzung der beteiligten Systeme und Werkzeuge. Der Beitrag zeigt auf, wie der offene Zugriff auf Feldgeräteinformation mittels Field Device Integration (FDI) und OPC UA bereits heute möglich ist. Nach einer Einordnung des Themas in den Kontext Industrie 4.0, Industrial Internet of Things und cyber-physische Systeme erfolgt eine genauere Betrachtung der Technologien FDI und OPC UA, bei der das FDI-Informationsmodell für OPC UA im Fokus steht. Abschließend wird der Nutzen anhand eines Demonstrators erklärt.

\section{SCHLAGWÖRTER FDI / OPC UA / IIOT / CPS}

\section{FDI and OPC UA -}

Key elements of Industry 4.0

A key element of the application of Industry 4.0 is the flexible networking of the relevant systems and tools. We show how the open access to field device information by means of Field Device Integration (FDI) and OPC UA is already possible. After positioning the topic in the context of Industry 4.0, the Industrial Internet of Things und cyber-physical systems, FDI und OPC UA technologies are considered in more detail, with a focus on the FDI information model for OPC UA. Finally, possible benefits are outlined on the basis of a demonstrator.

KEYWORDS field device integration / OPC UA / IIOT / CPS 
DANIEL GROSSMANN, Technische Hochschule Ingolstadt

SVEN SEINTSCH, Bilfinger Maintenance

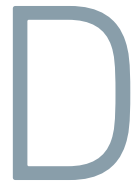

er Begriff Industrie 4.0 besitzt viele Facetten: Aus Sicht der Arbeitswelt stehen Themen wie Flexibilisierung der Arbeitszeiten aber ebenso verstärkte Mensch-Maschine-Kooperation im Vordergrund. Aus Sicht der Produktion spielen Themen wie horizontale Integration entlang der Wertschöpfungskette, vorausschauende Instandhaltung und Flexibilisierung der Produktion eine wichtige Rolle. Aus Engineeringsicht ist ebenfalls die horizontale Integration wichtig, diesmal aber im Sinne des nahtlosen Zusammenspiels aller Werkzeuge und Systeme entlang des Lebenszyklus von der Planung bis zum Rückbau [2].

\section{INDUSTRIE 4.0, INTERNET OF THINGS UND CYBER-PHYSISCHE SYSTEME}

Voraussetzung für die Umsetzung von Anwendungsfällen in allen genannten Facetten ist die umfassende Vernetzung von Werkzeugen, Komponenten und Systemen. Im Gegensatz zu heutigen Systemen, bei denen teils schon ein hoher Grad an Vernetzung zu finden ist, erfolgt die Vernetzung bei Industrie 4.0 anwendungsfallabhängig zur Laufzeit, das heißt, eine manuelle Kopplung mit hohem Engineeringaufwand entfällt, die Rollen Bereitsteller von Information und Nutzer von Information sind weniger starr zugeordnet und wechseln je nach Anwendungsfall. Von daher kann von einer Auflösung der (starren) Automatisierungspyramide gesprochen werden [1].

Grundsätzliche Konzepte für eine technische Umsetzung der genannten Vernetzung finden sich in Begriffen wie Industrial Internet of Things (IIoT) und cyber-physische Systeme (CPS) (Bild 1). Hinter beiden Begriffen steht der Ansatz, Entitäten der realen Welt (1, Things, physische Komponenten) über einen virtuellen Zwilling zu repräsentieren (2, Internet, Cyber). Anwendungen und Dienste greifen dann für ihre Umsetzung auf die virtuellen Repräsentanten zu, zum Beispiel, um deren Information zu vernetzen oder um deren Dienste zu überlagerten Diensten zu orchestrieren.
Die Basis einer jeden automatisierten Produktion bleiben die Sensoren und Aktoren. Diese Feldgeräte bilden die Schnittstelle zum technischen Prozess und sind somit als Things in jedem Industrie-4.0-Szenario vorhanden.

\section{FIELD DEVICE INTEGRATION UND INDUSTRIE 4.0}

Für die Integration von Feldgeräten wurde 2015 der internationale Standard Field Device Integration (FDI, IEC 62769) verabschiedet. Ziel von FDI ist die herstellerunabhängige Integration von Feldgeräten in Automatisierungssysteme. Dazu wird der Feldgerätetyp in Form eines FDI-Device-Packages beschrieben. Das DevicePackage enthält die Feldgeräteinformation, wie etwa einstellbare Parameter oder Diagnosen, Gerätefunktionen wie eine Selbsttestfunktion, sowie das User Interface zur Darstellung beziehungsweise Veränderung der Geräteparameter und zur Bedienung der Funktionen [3-5]. Umgesetzt werden diese Bestandteile des DevicePackages über die Technologien Electronic Device Description Language (EDDL) und .Net Windows Presentation Foundation (WPF) für optionale programmierte User Interface Plugins (Bild 2). Das FDI-Package wird von einem FDI-Host importiert und verarbeitet, sodass ein Benutzer Feldgeräte im Engineering offline konfigurieren und vorparametrieren kann. Die Konfiguration wird dann in der Inbetriebnahme online zum Feldgerät übertragen. Während des Betriebs kann die Diagnose des Feldgeräts sowie Anpassung der Parametrierung (etwa Tuning von Regelungsschleifen) erfolgen. Ein für Anwender, Geräte- und Systemhersteller wesentliches Ziel von FDI ist es, die nahtlose Integration von Feldgeräten unabhängig von Geräte-, System- oder Werkzeughersteller zu gewährleisten - ein Package, alle Werkzeuge [6].

Eine weitere wesentliche Endanwenderforderung ist der offene Zugang zu Geräteinformation. In der Regel ist dieser aktuell nur über proprietäre Leitsystemschnittstellen möglich. Ist dieser Zugang nicht gegeben oder zu eingeschränkt, bleibt noch die Option, einen parallelen Zugang zu den Feldgeräten zu schaffen. Dies lässt sich 


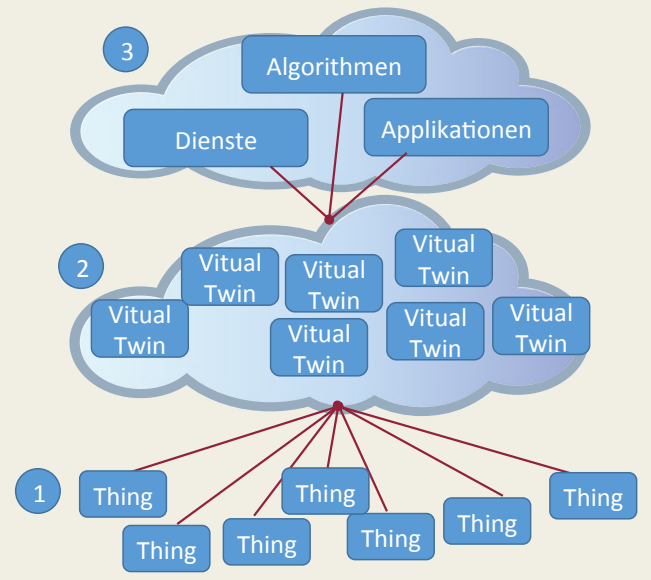

BILD 1: CPS, IoT Struktur

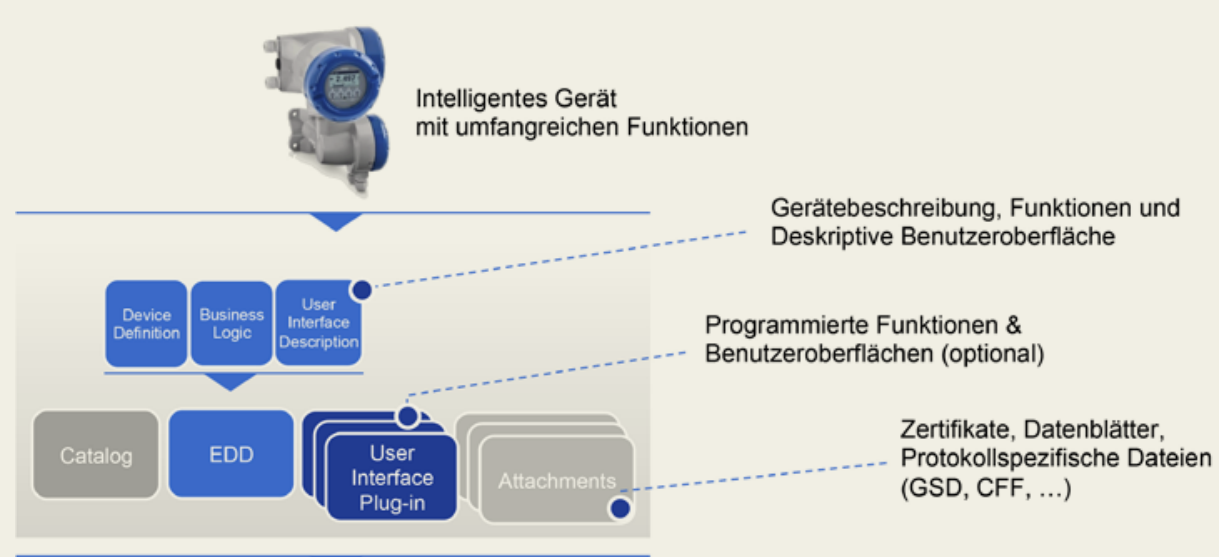

BILD 2: Inhalt des FDI

Device Packages
FDI Device Package, Eine Datei für den Nutzer.
Protokollspezifische Dateien (GSD, CFF, ...)

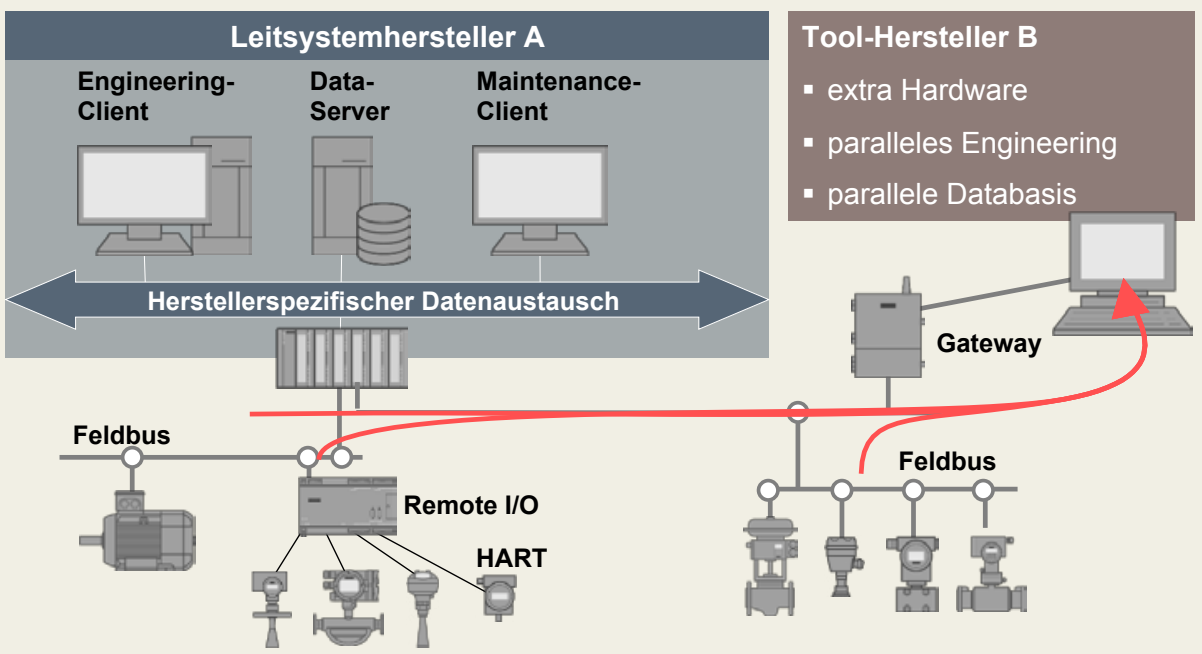

BILD 3: Paralleler Zugriff auf Feldgerätedaten

beispielsweise über Profibus Klasse 2 Master oder HartMultiplexer realisieren. In jedem Fall müssen aber Teile der Topologie des Automatisierungssystems ein zweites Mal geplant und verwaltet werden. Das schafft Aufwand und Redundanzen, die bei Änderungen zu Inkonsistenzen führen können (Bild 3). Um der wesentlichen Anwenderforderung nach offenem Zugang zu Gerätinformation gerecht zu werden, spezifiziert FDI eine herstellerunabhängige OPC-UA-Schnittstelle sowie das zugehörige Informationsmodell [7]. Über diesen standardisierten Zugang können nun Systeme, wie zum Beispiel Monitoring-Werkzeuge, auf Geräteinformation zugreifen und diese für ihren jeweiligen Anwendungsfall verarbeiten. Ein zusätzlicher Kommunikationskanal zu den Feldgeräten ist nicht erforderlich. Weitere Vorteile sind: 


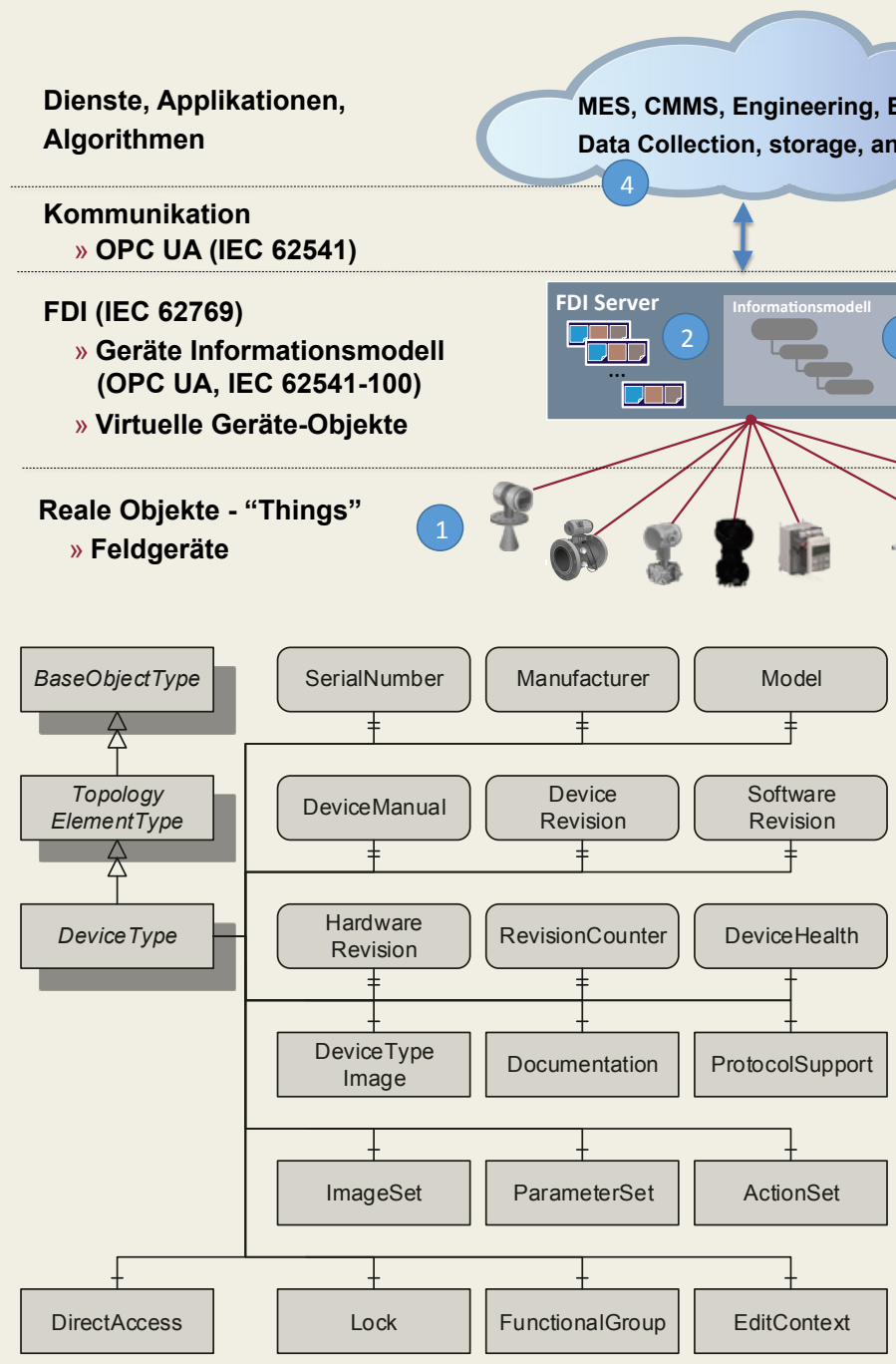

BILD 4: FDI im CPS und IoT Kontext

BILD 5: OPC UA DeviceType Definition

stellt der Server den Zugang zur Feldgeräteinformation zur Verfügung. OPC-UA-Clients (4) implementieren dann übergeordnete Algorithmen und bieten diese wiederum selbst als Dienste an. Somit ist es nur konsequent, dass FDI und OPC-UA als Technologien für die Umsetzung von Industrie 4.0 im Referenzarchitekturmodell für Industrie 4.0 (RAMI) empfohlen sind [8].

\section{FDI-OPC-UA-INFORMATIONSMODELL}

Im Rahmen der FDI-Spezifikationsarbeiten wurde als Zwischenergebnis das Device Integration (DI) OPC-UAInformationsmodell erarbeitet und als OPC-UA-Companion-Standard veröffentlicht [9]. Das DI-Modell bil- det die Basis für drei weitere Informationsmodelle: Analytical Device Integration (ADI), PLCopen und das Field-Device-Integration-Informationsmodell [7].

Das FDI-Informationsmodell legt fest, wie ein FDIServer Information darstellt und strukturiert. Es ist also gewissermaßen ein Vertrag, über den ein FDI-Client weiß, wo er welche Information im FDI-Server findet und wie diese aufgebaut ist, beispielsweise um einen Geräteparameter zu verändern. OPC-UA-Informationsmodelle sind objektorientiert aufgebaut und bestehen dementsprechend aus Objekten. Diese Objekte besitzen Eigenschaften (Properties), über die ein Objekt näher charakterisiert wird. Das kann zum Beispiel der Name eines Feldgeräteherstellers sein. Sollen Daten von Objekten abgebildet werden, so geschieht dies über Va- 

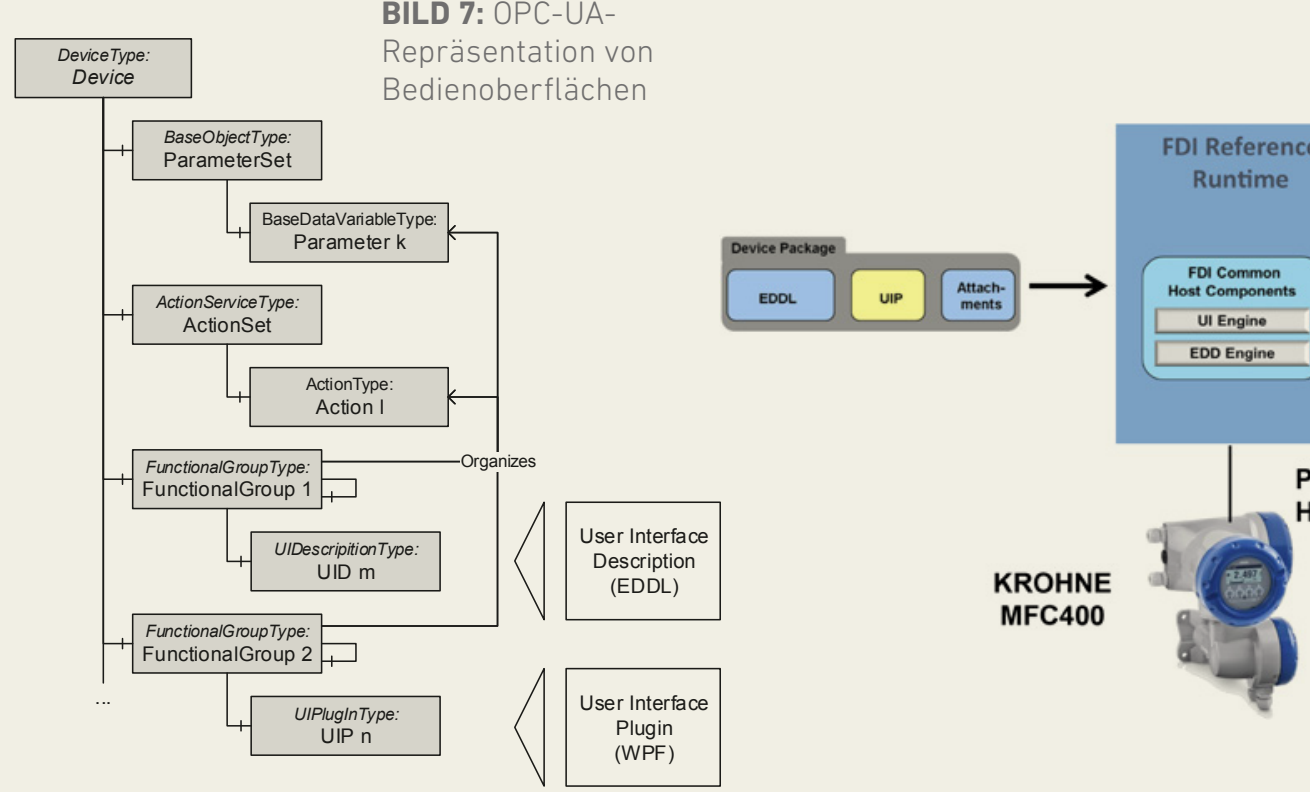

Runtime

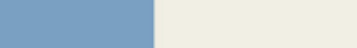

FDI Common

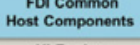

UI Engine

NE107

NE107
Status

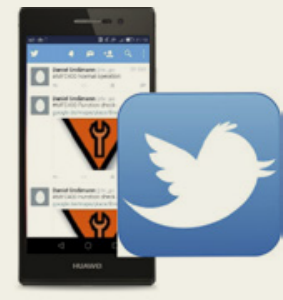

EDD Engine

Status

FDI Client

Assetmanagement
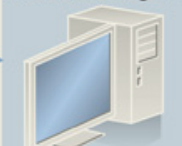

Hersteller $Y$

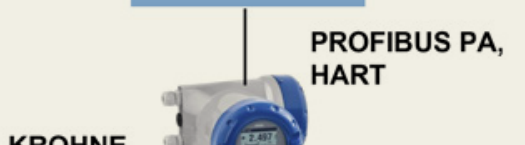

KROHNE MFC400

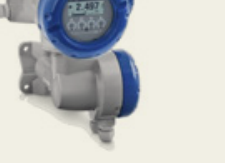

BILD 8: Struktur des

Demonstrators

riablen. Im Gegensatz zu Eigenschaften bilden Variablen eher veränderliche Daten ab, wie etwa den Temperaturwert eines Messumformers oder einen Feldgeräteparameter. Eigenschaften und Variablen können gelesen und geschrieben werden. Bei Wertänderung kann eine Benachrichtigung erfolgen.

Objekte können auch Methoden besitzen, über die sich Aktionen am Objekt auslösen lassen. Objekte können über Ereignisse generell Veränderungen melden, wie zum Beispiel die Änderung des internen Zustands. Durch Referenzen zwischen Objekten entsteht die Struktur des Informationsmodells. Ausgehend von einer Reihe von Basistypen, die dann über Vererbung zu spezifischen Typen hin verfeinert werden können, lassen sich damit Informationsmodelle erstellen. Kern des FDI-Informationsmodells ist der Typ DeviceType (Bild 5). Dieser Typ legt fest, wie ein Feldgerät im FDIInformationsmodell repräsentiert wird. Dazu besitzt der Typ eine Reihe von Eigenschaften, wie zum Beispiel die genaue Typbezeichnung (Model), die Seriennummer (SerialNumber) oder den Herstellernamen (Manufacturer). DeviceTypeImage enthält Bilder des Feldgeräts in verschiedenen Auflösungen, zum Beispiel für die Anzeige in einem Gerätekatalog. Documentation stellt die Dokumentation zum Gerät zur Verfügung. Zusätzliche Feldbusdateien, wie etwa eine GSD- oder GSDMLDatei, sind in ProtocolSupportFiles abgebildet.

Das ParameterSet enthält die Feldgerätedaten, also zum Beispiel Konfigurationsparameter und Status- und
Diagnoseinformation als OPC-UA-Variablen. Basis ist die in EDDL beschriebene Device Definition des FDIPackages (Bild 6). Die darin beschriebenen Daten werden im ParameterSet nach den Mapping-Regeln der FDI Specification Part 5 [7] gelistet.

FunctionalGroups bilden die Menüstruktur und Bedienoberflächen des Feldgeräts ab, das heißt sie spiegeln die im FDI-Package enthaltenen User Interface Descriptions (in EDDL) und User Interface Plugins (in WPF) wider (Bild 7). FunctionalGroups können weitere FunctionalGroups als Unterstruktur besitzen und referenzieren die in einer Bedienoberfläche genutzten Geräteparameter und -funktionen. Handelt es sich bei einer Bedienoberfläche um eine User Interface Description, dann ist diese als UIDescriptionType abgebildet. Dieses Objekt enthält die Beschreibung der Oberfläche im XML-Format. Handelt es sich um ein User Interface Plugin, dann wird dieses als UIPlugInType repräsentiert. Das Objekt stellt das programmierte User Interface Plugin als Byte-Array zur Verfügung.

Die in Bedienoberflächen genutzten Bilder sind im ImageSet enthalten. Die Feldgerätefunktionen sind im ActionSet definiert. Das Sperren eines Feldgeräteobjekts für den Schreibzugriff ist über das Locking realisiert. Der EditContext erlaubt es, Veränderungen zuerst in einer Kopie der Gerätedaten auszuführen. Für die direkte Kommunikation zum Feldgerät bietet DirectAccess entsprechende Funktionen an. 
Für Monitoring-Applikationen bietet der DeviceType die Variable DeviceHealth. Hier lässt sich der aktuelle Gerätezustand herstellerunabhängig abfragen. Der zurückgelieferte Wert enthält einen Zustandswert aus der Aufzählung der NE107 Zustände Out of Specification, Maintenance Required, Function Check und Failure [10]. Basis für die Berechnung des Zustandes ist eine EDDL-Methode, die im FDI-Device-Package zwingend enthalten sein muss.

\section{BEISPIELHAFTE UMSETZUNG}

Um die beschriebenen Mechanismen besser demonstrieren und nachvollziehen zu können, erfolgte eine Implementierung der OPC-UA-Server-Schnittstelle für die FDI Reference Runtime. Für den Workshop auf der Namur-Hauptsitzung 2015 wurde darauf aufbauend eine Demonstration erstellt: Für das Krohne-CoriolisMassedurchflussmessgerät MFC400 wurde jeweils ein FDI-Device-Package für Hart und Profibus-PA in die FDI Reference Runtime integriert, sodass das Informationsmodell des enthaltenen OPC-UA-Servers entsprechend erstellt wird. Eine OPC-UA-Client-Applikation greift dann auf die HealthStatus-Variable des Geräts im Informationsmodell $\mathrm{zu}$ und fragt diese ab. Neben einer einfachen Visualisierung des Zustands erfolgt die Weiterleitung als Tweet an Twitter. Darüber lässt sich der Gerätezustand in der Demonstration dann von jedem Smartphone aus verfolgen (Bild 8).

\section{AUSBLICK}

FDI und OPC UA bilden einen wichtigen Baustein für die Nutzung von Feldgeräteinformation in Industrie-4.0-Szenarien. Die Architektur beschreibt ein cyber-physisches System und fügt sich daher nahtlos in das Referenzarchitekturmodell für Industrie 4.0 ein. Der Zugang zu Feldgeräteinformation über die offene OPC-UA-Schnittstelle spart Aufwand und bildet die Basis für höherwertige Dienste im Sinne eine Industrie-4.0-Anwendung. Auf Umsetzungsseite sind bereits erste FDI-Hosts am Markt verfügbar. Im Sinne einer sinnvollen gestuften Markteinführung setzen diese die wesentliche Endanwenderforderung um: ein Package, alle Werkzeuge. Zusätzliche Produkte mit erweitertem Funktionsumfang sind angekündigt. Hier gilt es nun, darauf zu achten, dass diese Versionen die weitere Anwenderforderung anbieten, nämlich die beschriebene OPC-UA-Schnittstelle. Denn nur so lassen sich die vollen Potenziale von FDI und Industrie 4.0 tatsächlich nutzen.

\section{REFERENZEN}

[1] Promotorengruppe Kommunikation der Forschungsunion Wirtschaft - Wissenschaft (Hrsg.): Umsetzungsempfehlungen für das Zukunftsprojekt Industrie 4.0. April 2013.

[2] VDI/VDE Gesellschaft Mess- und Automatisierungstechnik (Hrsg.): Cyber-Physical Systems: Chancen und Nutzen aus Sicht der Automation. April 2013.

[3] Großmann D.: Offene Integrationsplattform für das FeldgeräteManagement. Sierke Verlag, August 2009, ISBN 978-3868440706 Uhlig, R.; Bruns, M.: Automatisierung von Chargenprozessen. Oldenbourg Verlag, 1995.

[4] IEC 62769-1: Field Device Integration (FDI), Technical Specification - Part 1: Overview. IEC, 2013.

[5] Großmann D.; Braun M.; Danzer B.; Riedl M.: Field Device Integration: Handbuch für die einheitliche Integrationstechnologie. VDE Verlag, 11/2013, ISBN 978-3800735136

[6] NAMUR Empfehlung 105: Anforderungen an die Integration von Feldbus-Geräten in Engineering-Tools für Feldgeräte, September 2008.

[7] IEC 62769-5: Field Device Integration (FDI), Technical Specification - Part 5: FDI Information Model. IEC, 2013.

[8] ZVEI: Das Referenzarchitekturmodell Industrie 4.0 (RAMI 4.0), 2015.

[9] IEC 62541-100 CDV: OPC UA Specification - Part 100, OPC UA for Devices, 2010

[10] NAMUR Empfehlung 107: Selbstüberwachung und Diagnose von Feldgeräten, 2006.

\section{AUTOREN}

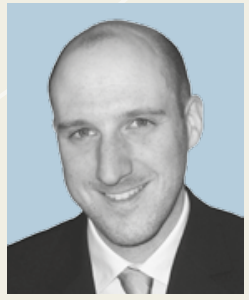

Prof. Dr.-Ing. DANIEL GROSSMANN (geb. 1977) ist Professor für Ingenieurinformatik und Datenverarbeitung an der TH Ingolstadt. Zu seinen Forschungsthemen zählen industrielle Kommunikation, Geräteintegration und Informationsmodellierung in der Automatisierungstechnik als Basis für Industrie 4.0, Cyber Physical Systems, und Industrial Internet of Things.

Technische Hochschule Ingolstadt,

Esplanade 10, D-85049 Ingolstadt,

Tel +49 (0) 84193482880 ,

E-Mail: Daniel.Grossmann@thi.de

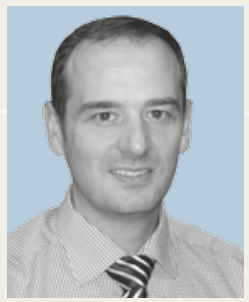

Dipl.-Ing. SVEN SEINTSCH leitet das Prüflabor der Bilfinger Maintenance $\mathrm{GmbH}$. Er ist Obmann des NAMURArbeitskreises 2.6 „Feldbus“. In der (IGR) e.V. Interessengemeinschaft Regelwerke Technik leitet er das Arbeitsfeld „Prüftechnik“. 